\title{
Uniqueness in First-Price Auctions with Affiliation
}

\author{
David McAdams*
}

June 8, 2004

The first-price auction has a unique monotone pure strategy equilibrium when there are $n$ symmetric risk-averse bidders having affiliated types and interdependent values.

KEYWORDs: First-price auction, uniqueness, affiliation, interdependent values, all-pay auction, cartels

A large and growing literature, both empirical and experimental, studies symmetric first-price auctions with the goal of testing whether observed behavior is consistent with equilibrium and/or of estimating the distribution of some unobservables taking equilibrium behavior as given. ${ }^{1}$ These papers

*I thank Nicola Persico for his generous help when this project was at a very early stage and Haluk Ergin, Sergei Izmalkov, and seminar participants at Arizona, Illinois, Pitt, and Rutgers for helpful conversations. Email: mcadams@mit.edu. Post: MIT Sloan School of Management, E52-448, 50 Memorial Drive, Cambridge MA 02142

${ }^{1}$ Hendricks, Pinkse, and Porter (2003) provide an overview of recent empirical work. For a survey of experimental work see e.g. Kagel (1995). 
focus on the symmetric equilibrium in monotone strategies demonstrated by Milgrom and Weber (1982); the possibility of other equilibria is effectively ignored. But if additional equilibria exist, then the conclusions of this literature would be jeopardized since they might be based on selecting the "wrong equilibrium." Uniqueness can be guaranteed if one is willing to assume independent private values but independence, especially, is overly strong in many applications. For instance, in a procurement setting, competing firms' costs may be driven by common input prices and hence positively correlated.

Despite the importance of this problem, very little progress has been made. Indeed, this is the first paper to prove uniqueness in first-price auctions in any environment with several bidders and non-independent types, including the case of affiliated private values. I show that there is a unique equilibrium when bidders are symmetric with affiliated types and interdependent values. The possibility of multiple equilibria in symmetric auctions is a real concern. Even in models with independent private values, the symmetric war of attrition has multiple equilibria, including a continuum of asymmetric equilibria [Nalebuff and Riley, (1985)]. Once the private values assumption is relaxed, the second-price auction and the open ascending-price auction also are well-known to have a continuum of asymmetric equilibria (in undominated strategies) [Milgrom (1981), Bikhchandani and Riley (1991)]. These asymmetric equilibria have a natural "winner's curse" intuition. When bidder 1 is more aggressive, this decreases bidder 2's expected value conditional on winning with any given bid, leading bidder 2 to bid less aggressively. In the same way bidder 2 being less aggressive leads bidder 1 to be more aggressive. This intuition would seem to apply to the first-price auction as well, 
making the problem of establishing uniqueness even more important.

Progress to this point has been limited to cases with independent private values or two bidders. Maskin and Riley (2003), Lebrun (1999), and Bajari (2001) prove uniqueness given independent private values and any number of asymmetric bidders. ${ }^{2}$ This literature also imposes restrictions on bidders' risk preferences: Maskin and Riley allow for non-increasing absolute riskaversion while Lebrun and Bajari require risk-neutrality. In contrast, my uniqueness result allows for affiliated types, interdependent values, and any sort of risk-aversion.

Lizzeri and Persico (2000) prove uniqueness of monotone pure strategy equilibrium ("MPSE") given affiliated types, interdependent values, and two asymmetric bidders. Environments with two bidders are special for several reasons, however, and insights drawn from this case may not apply in the more general context. For one thing, in two-bidder settings each bidder's bid schedule must clearly be continuous since any discontinuity would create a gap in the distribution of bids faced by the other bidder. The possibility of discontinuities in the several-bidder case vastly complicates the analysis since the set of active bidders can change from bid level to bid level. Even more disturbing, the Lipschitz condition needed to pin down local uniqueness of equilibrium can fail much more pervasively given several bidders. In the twobidder case as in the independent private values case, it is well understood that the Lipschitz condition can only fail at the lowest winning bid. Once

\footnotetext{
${ }^{2}$ Maskin and Riley (2003) and Lebrun (1999) each prove uniqueness in the case with a binding reserve price. When the reserve price is not binding, Lebrun (1999) proves uniqueness given that each bidder's valuation is drawn from one of two distributions, while Bajari (2001) extends this result to the general case.
} 
there are several (even symmetric) bidders, however, the Lipschitz condition can fail at higher bid levels as well. The key to my proof technique is to show that, in a symmetric environment, the problems usually associated with affiliation (discontinuities, failure of the Lipschitz condition, etc..) disappear if bidders' strategies are locally symmetric. Furthermore, the proof technique is constructive: first I show that all bidders make the same maximal bid, then employ the Fundamental Theorem of Differential Equations (FTODE) to uniquely pin down strategies that the bidders must follow at bid levels below the maximal bid. Since by uniqueness these strategies must be symmetric, I can continue to employ the FTODE to describe the entire equilibrium.

Like Lizzeri and Persico, this paper restricts attention to MPSE. Does this involve loss of generality? Fortunately, McAdams (2003) proves that every mixed-strategy equilibria is outcome-equivalent to some MPSE in a general asymmetric model, so proving uniqueness of MPSE proves uniqueness of mixed-strategy equilibrium. ${ }^{3}$

The rest of the paper is organized as follows. Section 1 lays out the model. Section 2 presents and proves the uniqueness result. Section 3 discusses extensions to all-pay auctions as well as implications for asymmetric firstprice auctions, including symmetric first-price auctions with a cartel.

\footnotetext{
${ }^{3}$ McAdams (2003)'s outcome-equivalence result is proven given a specific tie-breaking rule, the "priority rule" in which bidders are publicly assigned tie-breaking priorities before the bidding. Given the more standard "coin-flip rule" used here, McAdams shows that any equilibrium with zero probability of three-way ties is outcome-equivalent to a MPSE. While McAdams provides an example of a non-monotone equilibrium having positive probability of three-way ties in an asymmetric setting, I believe but have not proven that such examples can not exist in the symmetric setting in which this paper proves uniqueness of MPSE.
} 


\section{MODEL}

Information: Bidder types are one-dimensional random variables having joint density $f(\mathbf{t})$ on the unit cube $[0,1]^{n}$. For each subset $I \subset\{1, \ldots, n\}$, the conditional joint density will be denoted $f\left(\mathbf{t}_{I} \mid \mathbf{t}_{-I}\right)$ where $\mathbf{t} \equiv\left(t_{1}, \ldots, t_{n}\right), \mathbf{t}_{I} \equiv$ $\left(t_{i}: i \in I\right)$, and $-I \equiv\{1, \ldots, n\} \backslash I$. (Bold notation will be used throughout the paper to refer to vectors of types, bids, and strategies.)

(A1) $f(\cdot)$ continuously differentiable and positive on $[0,1]^{n}$.

(A2) Bidder types are affiliated, i.e. $f\left(\mathbf{t}^{\prime} \vee \mathbf{t}\right) f\left(\mathbf{t}^{\prime} \wedge \mathbf{t}\right) \geq f\left(\mathbf{t}^{\prime}\right) f(\mathbf{t})$ for all type profiles $\mathbf{t}^{\prime}, \mathbf{t}$, where $\mathbf{t}^{\prime} \vee \mathbf{t}, \mathbf{t}^{\prime} \wedge \mathbf{t}$ are their component-wise maximum and minimum, respectively.

Affiliation is a powerful form of positive correlation that allows us to establish that certain conditional expectations are non-decreasing. (See Milgrom and Weber (1982) for more detailed discussion.)

Bids and Payoffs: After learning its type, each bidder submits a bid $b_{i} \in$ OUT $\cup[r, \infty)$ where $r$ is the reserve price. Bidding is voluntary: a bidder who chooses not to participate "bids" OUT. If all bidders bid OUT, then the auction is cancelled. Otherwise the highest bidder wins the object, with ties broken by a coin-flip: if $k$ bidders each submit the highest bid, then each wins the object with probability $1 / k$. Bidder $i$ 's utility upon losing is zero and upon winning with bid $b$ has form $u_{i}\left(t_{i} ; \mathbf{t}_{-i} ; b\right)$. I make the following assumptions on utility: for all $i$,

(A3) $u_{i}$ twice continuously differentiable. 
(A4) $u_{i}$ strictly increasing in $t_{i}$, non-decreasing in $t_{j}$ for all $j \neq i$, and strictly decreasing in $b$

(A5) $\frac{\mathrm{d} u_{i}}{\mathrm{~d} b}$ non-decreasing in $\mathbf{t}$

(A6) $\frac{\mathrm{d} u_{i}}{\mathrm{~d} b}$ non-increasing in $b$

(A7) $u_{i}=u$ and $u\left(t_{i} ; \mathbf{t}_{-i} ; b\right)=u\left(t_{i} ; \mu\left(\mathbf{t}_{-i}\right) ; b\right)$ for any permutation $\mu$

(A8) $u\left(1 ; \mathbf{1} ; b^{h}\right)<0$ for some bid level $b^{h}<\infty$

The model is a special case of Reny and Zamir (2004). Thus, existence of a monotone pure strategy equilibrium is guaranteed. In particular, (A5) and (A8) are used to invoke Reny and Zamir. When utility takes the form $u\left(v\left(t_{i} ; \mathbf{t}_{-i}\right)-b\right),(\mathbf{A} 5)$ is satisfied if $u^{\prime \prime} \leq 0$, i.e. when bidders are riskneutral or risk-averse. (A8) implies that bidding $b^{h}$ or higher is a dominated strategy. The most notable additional assumptions are (A3) and (A6). (A3) is useful in verifying the Lipschitz condition in Section 2.2. (Somewhat weaker smoothness conditions would also suffice.) When utility takes the form $u\left(v\left(t_{i} ; \mathbf{t}_{-i}\right)-b\right),(\mathbf{A} 6)$ follows from $u^{\prime \prime} \leq 0$. Thus, my model is consistent with any sort of risk-aversion.

Let $b_{i}(\cdot)$ be a pure strategy and $b_{i}\left(t_{i}\right)$ the bid made by $i$-type $t_{i} . b_{i}(\cdot)$ is a monotone pure strategy iff $b_{i}\left(t_{i}^{\prime}\right) \geq b_{i}\left(t_{i}\right)$ for all $t_{i}^{\prime}>t_{i}$, where OUT is the lowest possible bid.

Definition (Monotone PURe Strategy Equilibrium): $\mathbf{b}^{*}(\cdot)$ is a monotone pure strategy equilibrium ("MPSE") if $b_{i}^{*}\left(t_{i}\right)$ is a best response to others' strategies $\mathbf{b}_{-i}^{*}(\cdot)$ for all types $t_{i}$.

Let $\mathbf{b}^{*}(\cdot)$ be a given MPSE. Common notation and shorthand: 
Inverse bid function: $\phi_{i}^{*}(b) \equiv \inf \left\{t_{i}: b_{i}^{*}\left(t_{i}\right) \geq b\right\}$. Any type less than $\phi_{i}^{*}(b)$ bids strictly less than $b$.

Winning event: $W_{i}(b) \equiv \times_{j \neq i}\left[0, \phi_{j}^{*}(b)\right] \equiv\left[\mathbf{0}, \boldsymbol{\phi}_{-i}^{*}(b)\right]$. Up to a zero measure set, $W_{i}(b)$ is the event in which bidder $i$ wins the object with bid $b$. (The analysis will show that $\phi_{i}^{*}(\cdot)$ are continuous in $b$.)

Winning probability: $p_{i}\left(t_{i}, b\right) \equiv \operatorname{Pr}_{\mathbf{t}_{-i} \mid t_{i}}\left(W_{i}(b) \mid t_{i}\right)$

Interim expected payoffs: $\Pi_{i}\left(t_{i}, b\right)$ is bidder $i$ 's expected payoff from bidding $b$ conditional on own type $t_{i}$ and others' equilibrium strategies:

$$
\begin{aligned}
\Pi_{i}\left(t_{i}, b\right) & \left.=p_{i}\left(t_{i}, b\right) E\left[u\left(t_{i} ; \mathbf{t}_{-i} ; b\right) \mid t_{i}, \mathbf{t}_{-i} \in W_{i}(b)\right)\right] \\
& =\int_{\mathbf{t}_{-i} \in W_{i}(b)} u\left(t_{i} ; \mathbf{t}_{-i} ; b\right) f\left(\mathbf{t}_{-i} \mid t_{i}\right) \mathrm{d} \mathbf{t}_{-i}
\end{aligned}
$$

Lowest winning bid: $\underline{b}_{i} \equiv \inf \left\{b: p_{i}\left(t_{i}, b\right)>0\right\}$. (By the full-support assumption, $p_{i}\left(t_{i}, b\right)>0$ for some $t_{i}$ implies $p_{i}\left(t_{i}, b\right)>0$ for all $t_{i}$.) By construction, $\underline{b} \equiv \max _{i} \underline{b}_{i}$ is the infimum of the support of $\max _{i} b_{i}^{*}\left(t_{i}\right)$.

\section{Preliminaries}

I extensively use an implication of Milgrom and Weber (1982) ("MW")'s Theorem 23. $A \subset X$ is a decreasing subset of $X$ when $x \in A, y \leq x \in X$ implies $y \in A$.

Corollary to MW Theorem 23: Suppose that $\mathbf{t}$ are affiliated, $X \subset$ $[0,1]^{k}$ is a lattice, and $A$ is a decreasing subset of $X$. Then $g: X \rightarrow \mathcal{R}$ non-decreasing implies that

$$
E[g(\mathbf{t}) \mid A] \leq E[g(\mathbf{t}) \mid X] \leq E[g(\mathbf{t}) \mid X \backslash A]
$$


If further $A \subsetneq X$ and $g$ strictly increasing, then

$$
E[g(\mathbf{t}) \mid A]<E[g(\mathbf{t}) \mid X]<E[g(\mathbf{t}) \mid X \backslash A]
$$

Before proceeding to the main result, I will rule out ties (Lemma 1) and show that bidders' strategies can only contain atoms at low enough bid levels.

Lemma 1 (No TIES): Suppose that $\operatorname{Pr}\left(\tilde{b} \geq \max _{i} b_{i}^{*}\left(t_{i}\right)\right)>0$. Then $\operatorname{Pr}\left(b_{i}^{*}\left(t_{i}\right)=b_{j}^{*}\left(t_{j}\right)\right)=0$ for all $i, j$.

Proof. Proven in McAdams (2003) for the more general case of asymmetric first-price auctions.

While obvious when there are just two bidders, Lemma 1 is much more difficult to prove once there are three or more bidders. In a three-bidder situation, for instance, bidder 1 wins with bid $b 33 \%$ of the time when both bidders 2,3 bid exactly $b, 50 \%$ when one of them bids exactly $b$ and the other bids less, and 100\% when both bid less. Raising its bid above $b$ will allow bidder 1 to raise these probabilities by $67 \%, 50 \%$, and $0 \%$, respectively, while decreasing its bid will lower them by 33\%,50\%, and 100\%. As long as bidders follow monotone strategies, however, this "probability wedge" does not lead a mass of bidder 1-types to find $b$ to be a best response.

Lemma 2 (No ATOMs): Suppose that $\operatorname{Pr}\left(\tilde{b} \geq \max _{i} b_{i}^{*}\left(t_{i}\right)\right)>0$ and $\tilde{b}>$ r. Then $\operatorname{Pr}_{t_{i}}\left(b_{i}^{*}\left(t_{i}\right)=\tilde{b}\right)=0$ for all $i$.

Proof. In the Appendix.

For an intuition, suppose that there were an atom at $\tilde{b}$. Since $\tilde{b}>r$, there can not be a gap in the distribution of winning bids just below $\tilde{b}$. (Otherwise, 
bidder $i$ would have bid lower.) So, some other bidder has a type that prefers to bid just less than $\tilde{b}$. This leads to a contradiction, however, since by slightly raising its bid that bidder can discontinuously raise its probability of winning.

\section{EqUiLibrium UNIQUENESS}

THEOREM 1: There is a unique monotone pure strategy equilibrium in the symmetric first-price auction (up to bids by a zero measure set of types).

The rest of this section provides the proof.

\subsection{Bidding behavior of the highest types}

Lemma 3 (Symmetry And Continuity at the top): $b_{i}^{*}(1-)=b_{i}^{*}(1) \equiv$ $\bar{b}$ for all $i$, where $b_{i}^{*}(1-) \equiv \lim _{\varepsilon \rightarrow 0} b_{i}^{*}(1-\varepsilon)$ denotes the left-limit.

Proof. It suffices to show that $b_{i}^{*}(1-)=b_{j}^{*}(1-)=\tilde{b}$ for all bidders $i, j$. If so, $\phi_{i}^{*}(\tilde{b})=1$ for all bidders $i$ so bidding $\tilde{b}$ guarantees that you will win the object. Thus, any bid greater than $\tilde{b}$ is strictly dominated and $b_{i}^{*}(1)=b_{i}^{*}(1-)$ for all $i$.

Now, suppose for the sake of contradiction that $\max _{i} b_{i}^{*}(1-)=b_{1}^{*}(1-) \equiv$ $\bar{b}_{1}>\bar{b}_{2} \equiv b_{2}^{*}(1-)$, implying that $\phi_{1}^{*}\left(\bar{b}_{2}\right)<1$. Since $\phi_{i}^{*}\left(\bar{b}_{1}\right)=1$ for all $i$, bidder 2-type $t_{2}=1$ can replicate bidder 1 -type $t_{1}=1$ 's payoff by bidding $\bar{b}_{1}$. (Bidding $\bar{b}_{1}$ allows both bidder 1 and bidder 2 to win with probability $100 \%$.) By revealed preference, then, $\Pi_{2}\left(1, \bar{b}_{2}\right) \geq \Pi_{1}\left(1, \bar{b}_{1}\right)$. For one thing, this requires that bidder 2 get positive payoff: $\operatorname{Pr}\left(W_{2}\left(\bar{b}_{2}\right) \mid t_{2}=1\right)>0$ and 


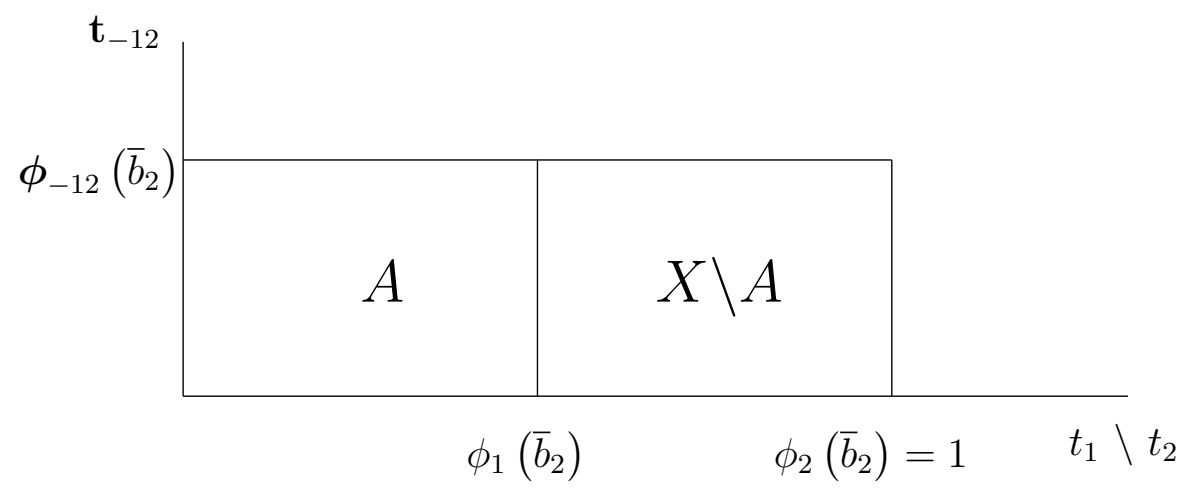

Figure 1: Lemma 3. Applying MW Theorem 23 to prove $\bar{b}_{1} \ngtr \bar{b}_{2}$.

$E\left[u\left(1 ; \mathbf{t}_{-2} ; \bar{b}_{2}\right) \mid W_{2}\left(\bar{b}_{2}\right)\right]>0$, where the relevant winning events are

$$
\begin{aligned}
& W_{1}\left(\bar{b}_{2}\right)=\left\{\mathbf{t}_{-1}: t_{2}<1 \text { and } \mathbf{t}_{-12}<\boldsymbol{\phi}_{-12}^{*}\left(\bar{b}_{2}\right)\right\} \\
& W_{2}\left(\bar{b}_{2}\right)=\left\{\mathbf{t}_{-2}: t_{1}<\phi_{1}^{*}\left(\bar{b}_{2}\right) \text { and } \mathbf{t}_{-12}<\boldsymbol{\phi}_{-12}^{*}\left(\bar{b}_{2}\right)\right\}
\end{aligned}
$$

On the other hand, $\Pi_{1}\left(1, \bar{b}_{1}\right)>\Pi_{2}\left(1, \bar{b}_{2}\right)$ since

$$
\begin{aligned}
\Pi_{1}\left(1, \bar{b}_{1}\right) & \geq \Pi_{1}\left(1, \bar{b}_{2}\right) \\
& =\operatorname{Pr}\left(W_{1}\left(\bar{b}_{2}\right) \mid t_{1}=1\right) E\left[u\left(t_{1} ; \mathbf{t}_{-1} ; \bar{b}_{2}\right) \mid t_{1}=1, W_{1}\left(\bar{b}_{2}\right)\right] \\
& \geq \operatorname{Pr}\left(W_{1}\left(\bar{b}_{2}\right) \mid t_{1}=1\right) E\left[u\left(t_{2} ; \mathbf{t}_{-2} ; \bar{b}_{2}\right) \mid t_{2}=1, W_{2}\left(\bar{b}_{2}\right)\right] \\
& >\operatorname{Pr}\left(W_{2}\left(\bar{b}_{2}\right) \mid t_{2}=1\right) E\left[u\left(t_{2} ; \mathbf{t}_{-2} ; \bar{b}_{2}\right) \mid t_{2}=1, W_{2}\left(\bar{b}_{2}\right)\right] \\
& =\Pi_{2}\left(1, \bar{b}_{2}\right)
\end{aligned}
$$

The first (weak) inequality is from bidder 1's revealed preference; the second (weak) inequality is from applying MW Theorem 23, where by symmetry one may think of $W_{2}\left(\bar{b}_{2}\right)$ as being a decreasing subset of $W_{1}\left(\bar{b}_{2}\right)$, as illustrated in Figure 1; and the last (strict) inequality uses that bidder 1 is more likely to win with bid $\bar{b}_{2}$ than bidder 2 . This is a contradiction. 


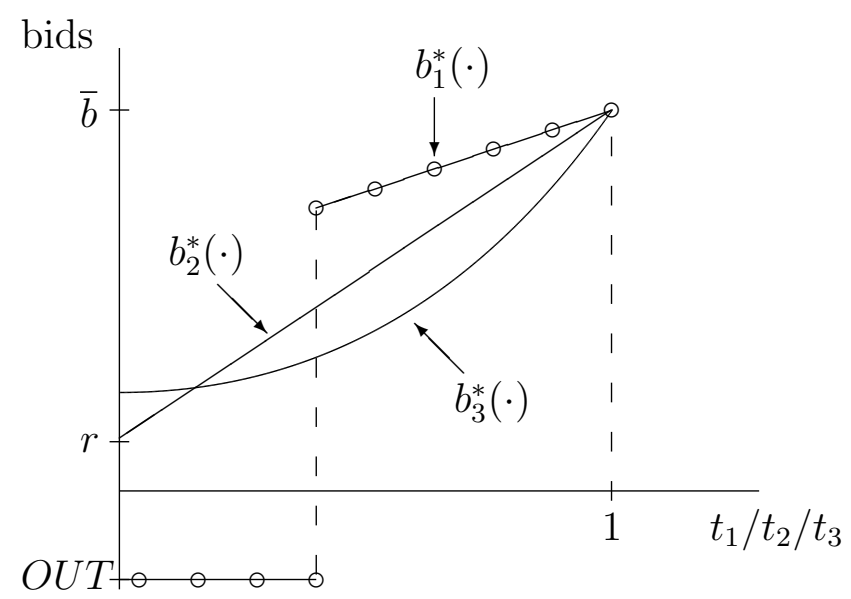

Figure 2: What has been proven: Section 2.1.

Figure 2 illustrates what has been proven so far about any MPSE (bidder 1's strategy is traced with circles): (i) all bidders make the same maximal bid $\bar{b}$ and (ii) each $b_{i}^{*}(\cdot)$ is continuous at $t_{i}=1$. Yet strategies need not coincide at lower types nor have I ruled out discontinuities at lower bid levels.

\subsection{Bidding behavior of the interior types}

By Lemma 3, all bidders' inverse bid functions are symmetric at $\bar{b}$, i.e. $\phi_{i}^{*}(\bar{b})=1$ for all $i$, and all bidders are active just below $\bar{b}$.

Definition (Active BIDDers): For $b>\underline{b}$, bidder $i$ is active just below $b$ if $b_{i}\left(\phi_{i}^{*}(b)-\right)=b$ and active at $b$ if $b_{i}\left(\phi_{i}^{*}(b)-\right)=b_{i}\left(\phi_{i}^{*}(b)+\right)=b . A(b-)$ denotes those bidders who are active just below $b$ and $A(b)$ those active at $b$. Bidder $i$ is active just below $b$ if it has some type $t_{i}(\varepsilon)$ that bids $b-\varepsilon$ for all small enough $\varepsilon>0$ while bidder $i$ is active at $b$ if it has some type $t_{i}$ that bids $b$ and $b_{i}\left(t_{i}\right)$ is continuous at $t_{i}$. 
Consider now the first-order conditions on each bidder's best responses. By Lemmas 1, 2 I may ignore ties and atoms at all bid levels $b>\underline{b}$. Furthermore, since I have restricted attention to monotone strategies the derivative of each bidder's inverse bid function, $\phi_{i}^{* \prime}(b)$, exists for a full measure set of bids. Thus, $\phi_{i}^{* \prime}\left(b_{i}\left(t_{i}\right)\right)$ exists for a full measure set of those types that bid greater than $\underline{b}, T_{i} \subset\left[\phi_{i}^{*}(\underline{b}), 1\right]$.

Let $b$ be a bid level such that $\phi_{i}^{*}(b) \in T_{i}$ for all $i$. Bidder $i$-type $\phi_{i}^{*}(b)$ chooses $b$ to maximize its objective

$$
b \in \arg \max _{\hat{b}} \int_{\mathbf{0}}^{\phi_{-i}^{*}(\hat{b})} u\left(\phi_{i}^{*}(b) ; \mathbf{t}_{-i} ; \hat{b}\right) f\left(\mathbf{t}_{-i} \mid \phi_{i}^{*}(b)\right) \mathrm{d} \mathbf{t}_{-i}
$$

The equilibrium first-order condition for each active bidder $i$ is

$$
\begin{aligned}
& 0=\sum_{j \neq i \in A(b)}\left\{\phi_{j}^{* \prime}(b) \int_{\mathbf{0}}^{\phi_{-i j}^{*}(b)} u\left(\phi_{i}^{*}(b) ; \phi_{j}^{*}(b), \mathbf{t}_{-i j} ; b\right) f\left(\phi_{j}^{*}(b), \mathbf{t}_{-i j} \mid \phi_{i}^{*}(b)\right) \mathrm{d} \mathbf{t}_{-i j}\right\} \\
& +\int_{0}^{\phi_{-i}^{*}(b)} \frac{\mathrm{d} u\left(\phi_{i}^{*}(b) ; \mathbf{t}_{-i} ; b\right)}{\mathrm{d} b} f\left(\mathbf{t}_{-i} \mid \phi_{i}^{*}(b)\right) \mathrm{d} \mathbf{t}_{-i} \\
& 0=\sum_{j \neq i \in A(b)}\left\{\phi_{j}^{* \prime}(b) a_{i j}\left(b, \phi^{*}(b)\right)\right\}+c_{i}\left(\phi^{*}(b)\right)
\end{aligned}
$$

where I use shorthand

$$
\begin{aligned}
a_{i j}\left(b, \phi^{*}(b)\right) & \equiv \int_{\mathbf{0}}^{\phi_{-i j}^{*}(b)} u\left(\phi_{i}^{*}(b) ; \phi_{j}^{*}(b), \mathbf{t}_{-i j} ; b\right) f\left(\phi_{j}^{*}(b), \mathbf{t}_{-i j} \mid \phi_{i}^{*}(b)\right) \mathrm{d} \mathbf{t}_{-i j} \\
c_{i}\left(\phi^{*}(b)\right) & \equiv \int_{\mathbf{0}}^{\phi_{-i}^{*}(b)} \frac{\mathrm{d} u\left(\phi_{i}^{*}(b) ; \mathbf{t}_{-i} ; b\right)}{\mathrm{d} b} f\left(\mathbf{t}_{-i} \mid \phi_{i}^{*}(b)\right) \mathrm{d} \mathbf{t}_{-i}
\end{aligned}
$$

$\left(\phi_{-i j}^{*}(b)\right.$ represents the vector $\left(\phi_{k}^{*}(b): k \neq i, j\right)$, and so on.) Notice that by the smoothness assumptions on utility $u$ and the density $f$, each $a_{i j}(b, \phi)$ is continuously differentiable in $b, \phi$ and each $c_{i}(\phi)$ is continuously differentiable in $\phi$. 
What about bid levels $b$ at which $\phi_{i}^{* \prime}(b)$ does not exist for some $i$ ? By continuity of the right-hand side of (2) in own type, the limit ${ }^{4}$ of first-order conditions of slightly lower types also applies to such types:

$$
\begin{aligned}
0= & \sum_{j \neq i \in A(b-)}\left\{\phi_{j}^{* \prime}(b-) \int_{\mathbf{0}}^{\phi_{-i j}^{*}(b)} u\left(\phi_{i}^{*}(b) ; \phi_{j}^{*}(b), \mathbf{t}_{-i j} ; b\right) f\left(\phi_{j}^{*}(b), \mathbf{t}_{-i j} \mid \phi_{i}^{*}(b)\right) \mathrm{d} \mathbf{t}_{-i j}\right\} \\
& +\int_{\mathbf{0}}^{\phi_{-i}^{*}(b)} \frac{\mathrm{d} u\left(\phi_{i}^{*}(b) ; \mathbf{t}_{-i} ; b\right)}{\mathrm{d} b} f\left(\mathbf{t}_{-i} \mid \phi_{i}^{*}(b)\right) \mathrm{d} \mathbf{t}_{-i}
\end{aligned}
$$

Thus, without loss I may continue as if the same system (2) applies to all bids in a neighborhood $(b-\varepsilon, b]$.

Suppose for the moment that the matrix $\boldsymbol{A}(b)=\left(a_{i j}(b, \boldsymbol{\phi})\right)$ is invertible. Then (2) can be written as a system of ordinary differential equations, as required by the Fundamental Theorem of Differential Equations ("FTODE"): 5

$$
\boldsymbol{\phi}^{* \prime}(b)=\boldsymbol{C}\left(\boldsymbol{\phi}^{*}(b)\right) \boldsymbol{A}^{-1}\left(b, \boldsymbol{\phi}^{*}(b)\right) \equiv \mathbf{g}\left(b, \boldsymbol{\phi}^{*}(b)\right)
$$

where each equation $g_{i}(b, \boldsymbol{\phi})$ is continuously differentiable in $b$ and $\boldsymbol{\phi}$. Consequently, $\mathbf{g}(b, \boldsymbol{\phi})$ satisfies the Lipschitz condition.

Of course, there is no guarantee that $\boldsymbol{A}\left(b, \boldsymbol{\phi}^{*}(b)\right)$ will be invertible in general. For $b>\underline{b}$, suppose that $\phi_{i}^{*}(b)=\phi^{*}(b)$ for all $i$ and let $\phi^{*}(b)$ be

\footnotetext{
${ }^{4}$ FOR REFEREES ONLY: It is conceivable that the left-derivatives $\phi_{j}^{* \prime}(b-)$ may not
} exist. Even so, system (3) must hold with respect to any left-accumulation point of $\left\{\phi_{j}^{* \prime}(b): j \neq i\right\}$ from below at $b$. By inspection of (2), note that each $\phi_{j}^{* \prime}(\tilde{b})$ is uniformly bounded over all $\tilde{b}$ in a neighborhood of $b$ :

$$
\phi_{j}^{* \prime}(\tilde{b})<2 \frac{c_{i}\left(\phi^{*}(b)\right)}{a_{i j}\left(b, \phi^{*}(b)\right)}<\infty
$$

Thus, such an accumulation point exists.

${ }^{5}$ See Theorem $2^{\prime}$ from Kolmogorov and Fomin (1975), p. 72. 
shorthand for a vector of types (of length $\geq 2$ ) all equal to $\phi^{*}(b)$. Then the matrix $\boldsymbol{A}\left(b, \boldsymbol{\phi}^{*}(b)\right)$ is invertible as it takes a particularly simple form: all diagonal entries are zero and all off-diagonal entries are equal and positive. Each $a_{i j}\left(b, \phi^{*}(b)\right)$ is positive because

$a_{i j}\left(b, \phi^{*}(b)\right)=E\left[u\left(t_{i} ; \mathbf{t}_{-i} ; b\right) \mid \mathbf{t}_{i j}=\boldsymbol{\phi}^{*}(b), \mathbf{t}_{-i j} \leq \boldsymbol{\phi}^{*}(b)\right] \operatorname{Pr}\left(\mathbf{t}_{-i j} \leq \boldsymbol{\phi}^{*}(b) \mid \mathbf{t}_{i j}=\boldsymbol{\phi}^{*}(b)\right)$

$E\left[u\left(t_{i} ; \mathbf{t}_{-i} ; b\right) \mid \mathbf{t}_{i j}=\boldsymbol{\phi}^{*}(b), \mathbf{t}_{-i j} \leq \boldsymbol{\phi}^{*}(b)\right] \geq E\left[u\left(t_{i} ; \mathbf{t}_{-i} ; b\right) \mid t_{i}=\phi^{*}(b), \mathbf{t}_{-i} \leq \boldsymbol{\phi}^{*}(b)\right]$

$E\left[u\left(t_{i} ; \mathbf{t}_{-i} ; b\right) \mid t_{i}=\phi^{*}(b), \mathbf{t}_{-i j} \leq \phi^{*}(b)\right] \operatorname{Pr}\left(\mathbf{t}_{-i} \leq \phi^{*}(b) \mid t_{i}=\phi^{*}(b)\right)>0$

(5) is a restatement of the definition of $a_{i j}\left(b, \phi^{*}(b)\right)$. (6) is an application of MW Theorem 23. (7) follows from the basic fact that bidders who win with positive probability must get positive expected utility. (See the argument surrounding equation (9) in the Appendix.)

Lemma 4 (Invertibility): Consider an $n \times n$ matrix $\boldsymbol{A}=\left(a_{i j}\right)$ in which $a_{i i}=0$ and $a_{i j}=a>0$ for all $i$ and $j \neq i$. Then $\boldsymbol{A}$ is invertible.

Proof. The proof is straightforward and so omitted.

By Lemma 3, all bidders are active just below $\bar{b}$ and $\phi_{i}^{*}(\bar{b})=\phi^{*}(\bar{b})=1$ for all $i$. Thus, $A\left(\bar{b}, \phi^{*}(\bar{b})\right)$ is invertible and, by continuity, $A\left(b, \boldsymbol{\phi}^{*}(b)\right)$ is invertible for all $b$ in a neighborhood $(\bar{b}-\varepsilon, \bar{b}]$. Thus, the FTODE implies that the equilibrium inverse bid functions are uniquely determined over this neighborhood. (Above $\bar{b}$ no bidder is active so the first-order conditions are not relevant.) Of course, uniqueness implies symmetry of this local solution 
since any asymmetric solution would lead to other asymmetric solutions when one permutes the bidders' identities. So, $\phi_{i}^{*}(b)=\phi_{j}^{*}(b)$ for all $i, j$ and $b \in$ $(\bar{b}-\varepsilon, \bar{b}]$. I show now that $\phi_{i}^{*}(b)=\phi_{j}^{*}(b)$ for all $i, j$ and $b>\underline{b}$. Suppose otherwise, that there exists $\tilde{b} \in(\underline{b}, \bar{b}-\varepsilon]$ such that (i) $\phi_{i}^{*}(b)=\phi_{j}^{*}(b)$ for all $i, j$ and all $b>\tilde{b}$ but (ii) $\phi_{i}^{*}(\tilde{b}-\partial) \neq \phi_{j}^{*}(\tilde{b}-\partial)$ for some bidders $i, j$ and arbitrarily small $\partial>0$.

By continuity of each $\phi_{i}^{*}(\cdot)$, (i) implies $\phi_{i}^{*}(\tilde{b})=\phi_{j}^{*}(\tilde{b})$ for all $i, j$. Repeating the argument of Lemma 3 , symmetry at $\tilde{b}$ implies that $b_{i}(\cdot)$ is continuous at $\phi_{i}^{*}(\tilde{b})$, so that all bidders are active just below $\tilde{b}$. Next, by repeating the FTODE argument above, there is a unique local solution to the system of $n$ first-order conditions just below $b$ so that, for some $\partial>0, \phi_{i}^{*}(b)=\phi_{j}^{*}(b)$ for all $i, j$ and all $b \in(\tilde{b}-\partial, \tilde{b}+\partial)$. But this contradicts (ii).

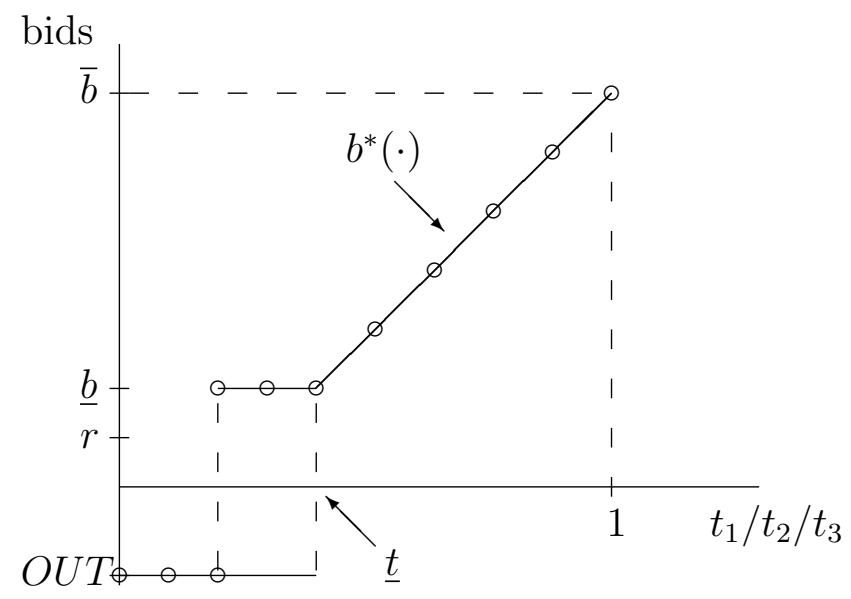

Figure 3: What has been proven: Sections 2.1-2.2.

Figure 3 illustrates what has been proven so far about any MPSE (bidder 1's strategy is again traced with circles). In addition to what I showed earlier, 
there exists type $\underline{t}$ such that (i) $b_{i}^{*}(t)=b^{*}(t)$ for all $i$ and all $t>\underline{t}$ and (ii) each bidder has zero probability of winning with any bid less than or equal to $\lim _{\varepsilon \rightarrow 0} b^{*}(\underline{t}+\varepsilon)$. In particular, $\underline{b}=b^{*}(\underline{t})$. Yet different equilibria may have different values for $\bar{b}, \underline{b}$, and/or $\underline{t}$. Also, Lemma 2 leaves open the case that there may be an atom in some bidder's strategy at $\underline{b}$ when $\underline{b}=r$, as depicted in Figure 3 for bidder 1.

\subsection{Bidding behavior of the lowest types}

Lemma 5 shows that all equilibria must have the same "starting condition" $(\underline{b}, \underline{t})$. Let $\underline{\mathbf{t}}_{I} \equiv\left(t_{i}=\underline{t}: i \in I\right)$ be shorthand for a vector of types all equal to $\underline{t}$.

Lemma 5 (Unique "Starting CONDition"): Define

$$
h(\underline{t}, \underline{b}) \equiv E\left[u\left(t_{i} ; \mathbf{t}_{-i} ; \underline{b}\right) \mid t_{i}=\underline{t}, \mathbf{t}_{-i} \leq \underline{\mathbf{t}}\right]
$$

Case I: $h(0, r) \geq 0$. In this case, $\underline{b} \geq r, \underline{t}=0$, and $\underline{b}$ is determined by $h(0, \underline{b})=0$. Case II $: h(0, r) \leq 0$. In this case, $\underline{b}=r, b_{i}\left(t_{i}\right)=$ OUT for all $t_{i}<\underline{t}$, and $\underline{t}$ is determined by $h(\underline{t}, r)=0$.

Proof. First, either $\underline{t}=0$ or $\underline{b}=r$. Suppose otherwise, that $\underline{t}>0$ and $\underline{b}>r$. $\underline{t}>0$ implies $\operatorname{Pr}\left(\underline{b} \geq \max _{i} b_{i}^{*}\left(t_{i}\right)\right)>0$. Thus, the proof of Lemma 2 implies that no bidder can bid $\underline{b}$ with positive probability. But by definition of $\underline{b}$, $b_{i}^{*}\left(t_{i}\right) \in\{O U T, \underline{b}\}$ for all $t_{i}<\underline{t}$, so that there is a gap in the distribution of winning bids below $\underline{b}$. This leads to a contradiction, however, since each bidder is strictly better off bidding $r$ rather than $\underline{b}$.

Next, $b_{i}^{*}\left(t_{i}\right)=O U T$ for all $i$ and all $t_{i}<\underline{t}$. The only case we need to check is $\underline{t}>0$ and $\underline{b}=r$. Lemma 1 implies that at most one bidder (say 


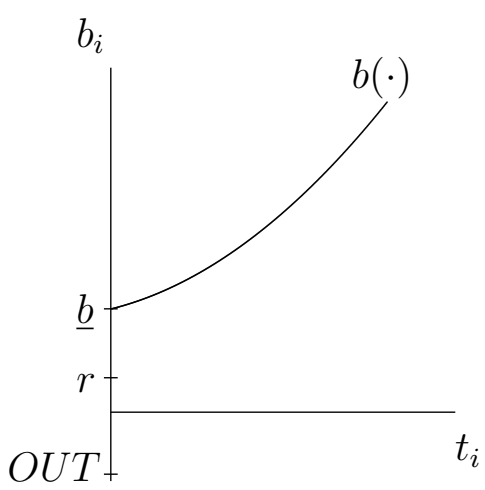

Figure 4: When $v(0 ; \mathbf{0})>r$, $\underline{t}=0$ and $\underline{b}=v(0 ; \mathbf{0})$.

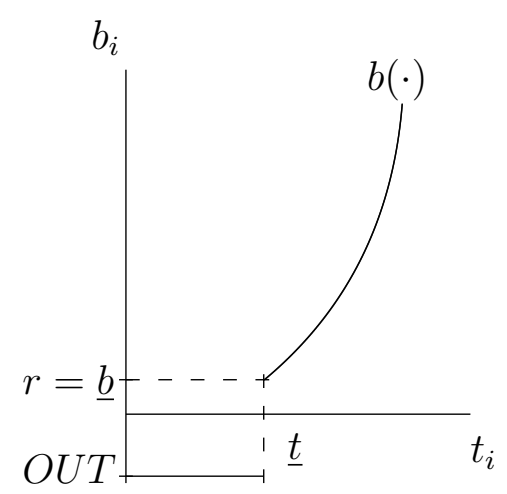

Figure 5: When $v(0 ; \mathbf{0})<r, \underline{b}=r$ and $E\left[v\left(t_{i} ; \mathbf{t}_{-i}\right) \mid t_{i}=\underline{t}, \mathbf{t}_{-i}<\underline{\mathbf{t}}\right]=r$.

bidder 1) bids $r$ with positive probability. But since bidder 2 is willing to bid OUT with any type $t_{2}<\underline{t}$ and is willing to bid more than $\underline{b}$ with any type $t_{2}>\underline{t}$, bidder 2 must get zero expected payoff given type $t_{2}=\underline{t}$ :

$$
E\left[u\left(t_{2} ; \mathbf{t}_{-2} ; \underline{b}\right) \mid t_{2}=\underline{t}, \mathbf{t}_{-2} \leq \underline{\mathbf{t}}\right]=0
$$

But this is also bidder 1's expected payoff from bidding $\underline{b}$ with type $\underline{t}$. Finally, by MW Theorem 23, bidder 1's expected payoff from bidding $\underline{b}$ is strictly increasing in $t_{1}$, so that all types $t_{1}<\underline{t}$ get negative expected payoff, a contradiction. (To apply MW, let $X=\left\{t_{1}, t_{1}^{\prime}\right\} \times\left(\times_{j \neq 1}[0, \underline{t}]\right)$ and $A=t_{1} \times$ $\left(\times_{j \neq 1}[0, \underline{t}]\right)$ where $t_{1}<t_{1}^{\prime}$.)

Consider Case I: $h(0, r) \geq 0$. Since $h(\underline{t}, \underline{b})$ is non-decreasing in $\underline{t}$ and strictly decreasing in $\underline{b}, h(\underline{t}, \underline{b})=0$ requires that $\underline{b}>r$ (and hence that $\underline{t}=0$ ). Now consider Case II: $h(0, r) \leq 0 . h(\underline{t}, \underline{b})=0$ requires $\underline{t}>0$ (and hence $\underline{b}=r)$.

Figures 4, 5 illustrate the two cases of Lemma 5 given risk-neutral bidders, 


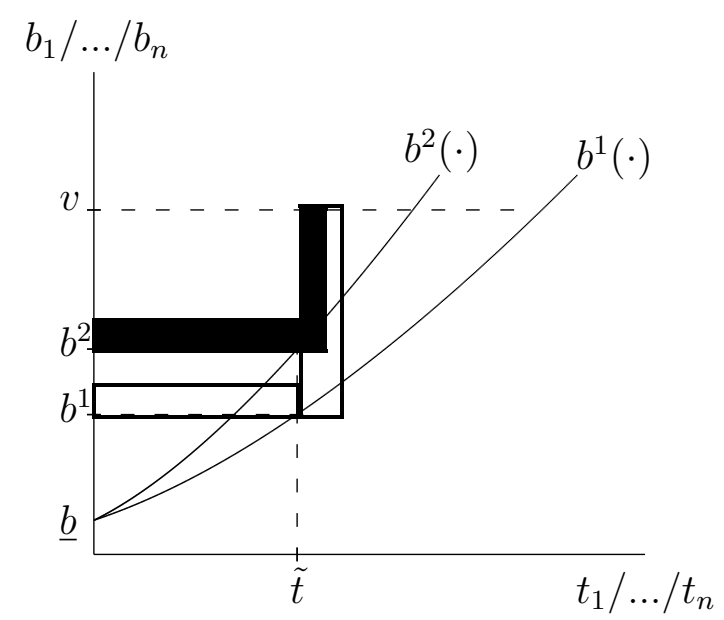

Figure 6: Graphical intuition why equilibria can not be ordered.

i.e. $u\left(t_{i} ; \mathbf{t}_{-i} ; b\right)=v\left(t_{i} ; \mathbf{t}_{-i}\right)-b$. Note that Case $\mathrm{I}$ is equivalent to $v(0 ; \mathbf{0}) \geq r$ while Case II is equivalent to $v(0 ; \mathbf{0}) \leq r$.

\subsection{Uniqueness}

Consider Case I in which the reserve price is not binding. (Case II proceeds in a similar way.) The previous steps have shown that any equilibrium must be in symmetric, continuously differentiable, and strictly monotone strategies with unique "starting condition" $b_{i}^{*}(0)=\underline{b}$ for all $i$ (determined by the relationship $u(0 ; \mathbf{0} ; \underline{b})=0)$ and "final condition" $b_{i}^{*}(1)=\bar{b}$ for all $i$. The question remains whether there might be more than one final condition consistent with equilibrium.

Suppose that $\mathbf{b}^{* 1}(\cdot)=\left(b^{* 1}(\cdot), \ldots, b^{* 1}(\cdot)\right)$ and $\mathbf{b}^{* 2}(\cdot)=\left(b^{* 2}(\cdot), \ldots, b^{* 2}(\cdot)\right)$ are two such symmetric equilibria with $\bar{b}^{2}>\bar{b}^{1}$, where I have added superscripts to all notation to distinguish the two equilibria. By continuity and local 
uniqueness of the solutions to system $(2), b^{2}(\cdot)$ and $b^{1}(\cdot)$ can never cross at any bid level $b>\underline{b}$. So, the equilibria are ordered with $b^{2}(t)>b^{1}(t)$ for all $t>0$.

For given type $\tilde{t}$, let $b^{2} \equiv b^{2}(\tilde{t})$ and $b^{1} \equiv b^{1}(\tilde{t})$, and define shorthand $\tilde{\mathbf{t}}_{I}=$ $\left(t_{i}=\tilde{t}: i \in I\right)$ for all $I \subset\{1, \ldots, n\}$. Consider bidders' first-order conditions in both equilibria given type $\tilde{t}$. Graphically for the risk-neutral case, the trade-off associated with bidding slightly higher is captured in Figure 6. In each equilibrium: the extra expected payment made when the bidder would have won anyway is the area of the horizontal rectangle; the extra surplus from the marginal event in which the bidder now wins is the area of the vertical rectangle. ( $v$ in the figure is shorthand for the bidder's expected value conditional on tying. Rectangles corresponding to equilibrium 2 are filled.) For a given increase $\triangle$ in the bid, the two horizontal rectangles have the same area. Since $b^{2}(\tilde{t})>b^{1}(\tilde{t})$, the vertical rectangle for equilibrium 1 has more height. Since the bidder must be indifferent to raising its bid in each equilibrium, the vertical and horizontal areas must be the same in each equilibrium, implying that the vertical rectangle for equilibrium 1 has less width. (This is not true in Figure 6.) That is to say, $b^{* 1 \prime}(\tilde{t})>b^{* 2 \prime}(\tilde{t})$ for all $\tilde{t}>0$. But this contradicts the presumption that $b^{* 1}(\tilde{t})<b^{* 2}(\tilde{t})$ since $b^{* 1}(0)=b^{* 2}(0)$ and these bid functions are continuous.

More formally, simplifying (2) given symmetry of all strategies by bidders 
$j \neq i$ in both equilibria, bidder $i$ 's first-order conditions are:

$$
\begin{aligned}
& 0=(n-1)\left\{\phi^{* 2 \prime}\left(b^{2}\right) \int_{\mathbf{0}}^{\tilde{\mathbf{t}}_{-i j}} u\left(\tilde{t} ; \tilde{t}, \mathbf{t}_{-i j} ; b^{2}\right) f\left(\tilde{t}, \mathbf{t}_{-i j} \mid t_{i}=\tilde{t}\right) \mathrm{d} \mathbf{t}_{-i j}\right\} \\
& +\int_{\mathbf{0}}^{\tilde{\mathbf{t}}_{-i}} \frac{\mathrm{d} u\left(\tilde{t} ; \mathbf{t}_{-i} ; b^{2}\right)}{\mathrm{d} b} f\left(\mathbf{t}_{-i} \mid t_{i}=\tilde{t}\right) \mathrm{d} \mathbf{t}_{-i} \\
& 0=(n-1)\left\{\phi^{* 1 \prime}\left(b^{1}\right) \int_{\mathbf{0}}^{\tilde{\mathbf{t}}_{-i j}} u\left(\tilde{t} ; \tilde{t}, \mathbf{t}_{-i j} ; b^{1}\right) f\left(\tilde{t}, \mathbf{t}_{-i j} \mid t_{i}=\tilde{t}\right) \mathrm{d} \mathbf{t}_{-i j}\right\} \\
& +\int_{0}^{\tilde{\mathbf{t}}_{-i}} \frac{\mathrm{d} u\left(\tilde{t} ; \mathbf{t}_{-i} ; b^{1}\right)}{\mathrm{d} b} f\left(\mathbf{t}_{-i} \mid t_{i}=\tilde{t}\right) \mathrm{d} \mathbf{t}_{-i}
\end{aligned}
$$

Since $u$ is strictly decreasing in $b$ and $\mathrm{d} u / \mathrm{d} b$ is non-increasing in $b$, it must be that $\phi^{* 2 \prime}\left(b^{2}\right)>\phi^{* 1 \prime}\left(b^{1}\right)$ which again implies $b^{* 1 \prime}(\tilde{t})>b^{* 2 \prime}(\tilde{t})$ and a contradiction. Thus, there can be only one equilibrium. This completes the proof of Theorem 1.

\section{EXTENSIONS}

This paper has proven that symmetric first-price auctions have a unique monotone pure strategy equilibrium. It is worth noting, however, that its results and techniques extend to some other auction environments.

Asymmetric first-price auctions: Consider a first-price auction with $K$ "classes" of bidders, $I_{1}, \ldots, I_{K}$, where bidders within each class of symmetric. ${ }^{6}$ It is straightforward to adapt the techniques developed here to show that all

\footnotetext{
${ }^{6}$ That is to say, both the joint density function and bidders' utility functions treat bidders from the same class symmetrically. For all $k$ and permutations $\mu$ : (i) $f\left(\mathbf{b}_{I_{k}}, \mathbf{b}_{-I_{k}}\right)=$ $f\left(\mu\left(\mathbf{b}_{I_{k}}\right), \mathbf{b}_{-I_{k}}\right)$; (ii) $u_{i}\left(t_{i} ; \mathbf{t}_{I_{k} \backslash i} ; \mathbf{t}_{-I_{k}}\right)=u_{i}\left(t_{i} ; \mu\left(\mathbf{t}_{I_{k} \backslash i}\right) ; \mathbf{t}_{-I_{k}}\right)$ for all $i \in I_{k}$; and (iii) $u_{i}\left(t_{i} ; \mathbf{t}_{I_{k}} ; \mathbf{t}_{-I_{k}}\right)=u_{i}\left(t_{i} ; \mu\left(\mathbf{t}_{I_{k}}\right) ; \mathbf{t}_{-I_{k}}\right)$ for all $i \notin I_{k}$.
} 
monotone pure strategy equilibria must be symmetric with respect to classes, i.e. $b_{i}^{*}(\cdot)=b_{j}^{*}(\cdot)$ whenever $i, j$ belong to the same class.

First-price auctions with a cartel: More specifically, consider the case with two classes in which one class includes $n-1$ out of $n$ bidders. While specialized, models of this type have proven useful to study bidding in situations in which some group of bidders acting together as "bidder $n$ " have formed a cartel [for example, see Marshall et al (1994)]. Clearly, $b_{n}^{*}(1) \leq b_{1}^{*}(1)$ since otherwise bidder $n$ could still win all of the time with a bid less than $b_{n}^{*}(1)$. Suppose further that, under extra assumptions, one could prove that $b_{n}^{*}(1) \geq b_{1}^{*}(1)$. Then every equilibrium would have to have "final condition" of the form $b_{i}^{*}(1)=\bar{b}$ for all $i$ as in my analysis of the symmetric case. Furthermore, the matrix $\boldsymbol{A}$ introduced in Section 2.2 is invertible as it still takes a simple form:

$$
\left(\begin{array}{ccccc}
0 & a & \ldots & a & c \\
a & 0 & \ldots & a & c \\
\ldots & \ldots & \ldots & \ldots & \ldots \\
a & a & \ldots & 0 & c \\
b & b & \ldots & b & 0
\end{array}\right)
$$

(The matrix has 0 on the diagonal, $b>0$ in the $n$th row, $c>0$ in the $n$ th column, and $a>0$ elsewhere.) Thus, the Lipschitz condition is satisfied whenever bidders $1, \ldots, n-1$ are locally symmetric and one can "unwind" the equilibrium as in Section 2.2 to show that there is at most one equilibrium consistent with each possible $\bar{b}$. Finally, one can extend the arguments of Section 2.4 to show that the equilibrium is unique.

All-pay auctions: The first-price auction has the property that bidders get 
the same utility from losing as from not participating. This property is not essential to my analysis. What is essential is that each bidder's payoff does not depend on others' bids (except insofar as others' bids determine whether that bidder wins the object). Suppose that bidders have different utilities from winning and losing the object, $u_{i}^{W}\left(t_{i} ; \mathbf{t}_{-i} ; b_{i} ; \mathbf{b}_{-i}\right)$ and $u_{i}^{L}\left(t_{i} ; \mathbf{t}_{-i} ; b_{i} ; \mathbf{b}_{-i}\right)$. The analysis depends specifically on the fact that (i) $u_{i}^{W}, u_{i}^{L}$ each do not depend on $\mathbf{b}_{-i}$, (ii) $u_{i}^{W}$ is strictly decreasing in $b_{i}$ while $u_{i}^{L}$ is non-increasing in $b_{i}$, and (iii) $u_{i}^{W}\left(t_{i} ; \mathbf{t}_{-i} ; b_{i}\right)-u_{i}^{L}\left(t_{i} ; \mathbf{t}_{-i} ; b_{i}\right)$ is strictly increasing in $t_{i}$ and non-decreasing in $t_{-i}$. Among other places, (i) is important in the proof that all bidders submit the same maximal bid, (ii) in the proof that there are no atoms, and (iii) pervasively in all applications of Milgrom Weber (1982)'s affiliation tools.

In the all-pay auction, losers pay their own bid which does not depend on bidders' types. Thus, (i,ii,iii) are satisfied and the proof of Theorem 1 implies that the all-pay auction has a unique monotone pure strategy equilibrium. ${ }^{7}$ Yet whether the all-pay auction has non-monotone equilibria remains open (even in the two-bidder case) so the question of uniqueness remains partially unresolved.

Unlike Lizzeri and Persico (2000) ("LP"), however, my analysis does not apply to "exotic" auctions where (for instance) bidders' payments are an average of the highest and second-highest bids. This difference relates to a more fundamental distinction between my approach and LP's. Ultimately,

\footnotetext{
${ }^{7}$ To apply to the all-pay auction, the proof of Lemma 3 and the expression for the first-order equations must be modified slightly to reflect the fact that each bidder always pays its bid. But this does not change the argument in any substantive way.
} 
LP exploits that $u_{i}^{W}$ and $u_{i}^{L}$ are, respectively, strictly increasing and nondecreasing in bidder $i$ 's own type whereas I exploit that $u_{i}^{W}-u_{i}^{L}$ is strictly increasing in own type and non-decreasing in others' types.

\section{APPENDIX FOR PROOFS}

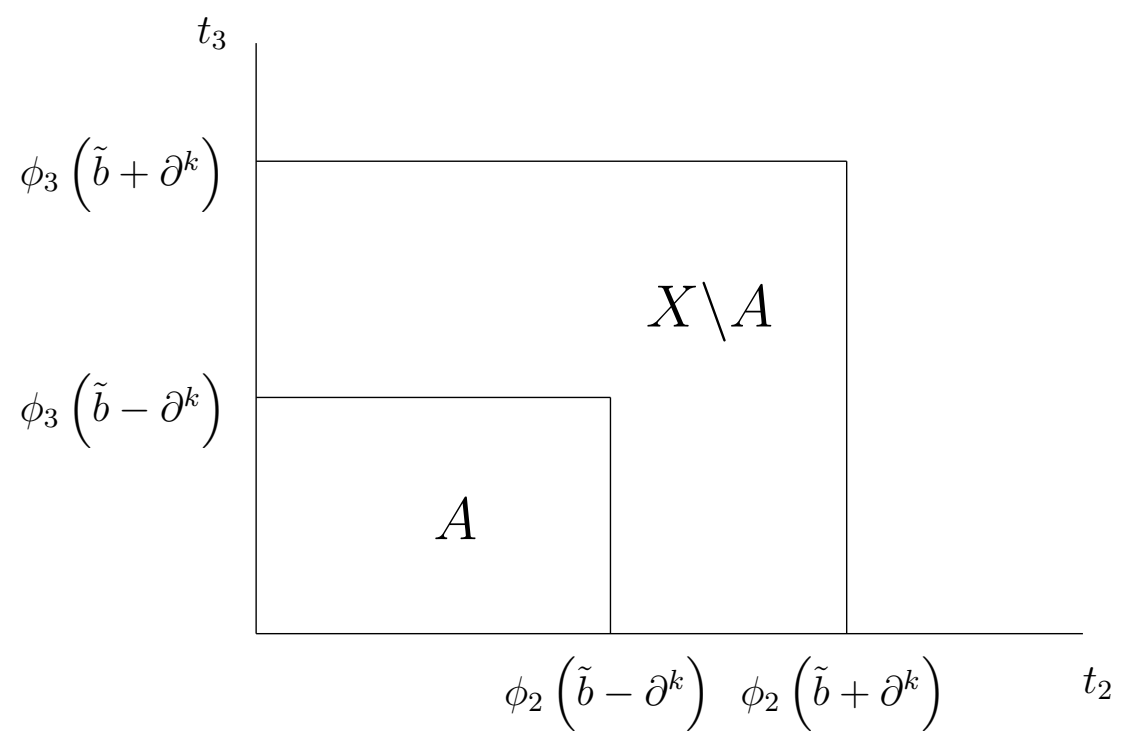

Figure 7: Lemma 2. Applying MW Theorem 23 to rule out atoms.

Proof of Lemma 2: Suppose that $\operatorname{Pr}\left(b_{i^{*}}^{*}\left(t_{i^{*}}\right)=\tilde{b}\right)>0$ for some $i^{*}$ with $\tilde{b}>r$. First, there can't be a gap in the distribution of $\max _{j \neq i^{*}} b_{j}^{*}\left(t_{j}\right)$ below $\tilde{b}$, else bidder $i^{*}$ would prefer to bid less than $\tilde{b}$. Thus, there exists a bidder $j^{*} \neq i^{*}$ with a sequence of types $\left\{t_{j^{*}}^{k}\right\}^{k=1,2, \ldots}$ such that $b_{j^{*}}^{*}\left(t_{j^{*}}^{k}\right)=\tilde{b}-\partial^{k}$ where the sequence $\partial^{k} \searrow 0$. Furthermore, $\left\{\partial^{k}\right\}^{k=1,2 \ldots}$ can be chosen so that $\lim _{k \rightarrow \infty} \operatorname{Pr}\left(\max _{i \neq j^{*}} b_{i}\left(t_{i}\right)=\tilde{b}-\partial^{k}\right)=0$. That is to say, even though some bidder other than $j^{*}$ might have a mass of types bidding exactly $\tilde{b}-\partial^{k}$, we 
may choose $\tilde{b}-\partial^{k}$ so that such atoms disappear. In the limit as $k \rightarrow \infty$, then, bidder $j^{*}$-type $t_{j^{*}}^{k}$ 's payoffs from bidding $\tilde{b}-\partial^{k}$ converges to what it would be if such atoms were ignored. So,

$$
\begin{aligned}
0 & \geq \lim _{k \rightarrow \infty} E\left[u\left(t_{j^{*}} ; \mathbf{t}_{-j^{*}} ; \tilde{b}\right) \mid t_{j^{*}}=t_{j^{*}}^{k}, \mathbf{t}_{j^{*}}: \max _{k \neq j^{*}} b_{k}^{*}\left(t_{k}\right) \in\left(\tilde{b}-\partial^{k}, \tilde{b}+\partial^{k}\right)\right] \\
& \geq \lim _{k \rightarrow \infty} E\left[u\left(t_{j^{*}} ; \mathbf{t}_{-j^{*}} ; \tilde{b}\right) \mid t_{j^{*}}=t_{j^{*}}^{k}, \mathbf{t}_{-j^{*}} \in W_{j^{*}}\left(\tilde{b}-\partial^{k}\right)\right] \\
& =\lim _{k \rightarrow \infty} E\left[u\left(t_{j^{*}} ; \mathbf{t}_{-j^{*}} ; \tilde{b}-\partial^{k}\right) \mid t_{j^{*}}=t_{j^{*}}^{k}, \mathbf{t}_{-j^{*}} \in W_{j^{*}}\left(\tilde{b}-\partial^{k}\right)\right] \\
& \equiv \lim _{k \rightarrow \infty} \lambda^{k}
\end{aligned}
$$

The first inequality follows from the fact that each type $t_{j^{*}}^{k}$ must prefer not raising its bid by $2 \partial_{k}$ from $b_{j^{*}}^{*}\left(t_{j^{*}}^{k}\right)=\tilde{b}-\partial^{k}$ to $\tilde{b}+\partial^{k}$. The second inequality follows from an application of MW Theorem 23: $X=W_{j^{*}}\left(\tilde{b}+\partial^{k}\right)$ is a lattice, $A=W_{j^{*}}\left(\tilde{b}-\partial^{k}\right)$ is a decreasing subset of $X$, and $X \backslash A=$ $\left\{\mathbf{t}_{j^{*}}: \max _{k \neq j^{*}} b_{k}^{*}\left(t_{k}\right) \in\left(\tilde{b}-\partial^{k}, \tilde{b}+\partial^{k}\right)\right\}$. (Figure 7 illustrates this step given three bidders and $j^{*}=1$.) Consequently,

$$
\lim _{k \rightarrow \infty} \Pi_{j^{*}}\left(t_{j^{*}}^{k}, b_{j^{*}}^{*}\left(t_{j^{*}}^{k}\right)\right) \leq \lim _{k \rightarrow \infty} \lambda^{k} \operatorname{Pr}\left(W_{j^{*}}\left(b_{j^{*}}^{*}\left(t_{j^{*}}^{k}\right)\right) \mid t_{j^{*}}^{k}\right) \leq 0
$$

On the other hand, fix $K$ and let $0<f_{\text {low }} \leq f_{\text {high }}<\infty$ be bounds on the density function. (Recall that $f(\mathbf{t})$ is assumed to be continuous and strictly positive over $\mathbf{t} \in[0,1]^{n}$.) Consider $k>K$ :

$$
\operatorname{Pr}\left(W_{j^{*}}\left(b_{j^{*}}^{*}\left(\left(t_{j^{*}}^{K}\right)\right) \mid t_{j^{*}}^{k}\right) \geq\left(\frac{f_{\text {low }}}{f_{\text {high }}}\right)^{n-1} \operatorname{Pr}\left(W_{j^{*}}\left(b_{j^{*}}^{*}\left(\left(t_{j^{*}}^{K}\right)\right) \mid t_{j^{*}}^{K}\right)\right.\right.
$$

Furthermore, by MW Theorem $23 E\left[u\left(t_{j^{*}} ; \mathbf{t}_{-j^{*}} ; b\right) \mid W_{j^{*}}(b)\right]$ is strictly increasing in $t_{j^{*}}$ for all $b$. Thus, type $t_{j^{*}}^{k}$ 's equilibrium payoff is positive if type $t_{j^{*}}^{K}$ wins with positive probability and, more important for the present purpose, type $t_{j^{*}}^{k}$ 's equilibrium payoff is bounded away from zero if type $t_{j^{*}}^{K}$ gets 
positive payoff:

$$
\begin{aligned}
\Pi_{j^{*}}\left(t_{j^{*}}^{k}, b_{j^{*}}^{*}\left(t_{j^{*}}^{k}\right)\right) & \geq \Pi_{j^{*}}\left(t_{j^{*}}^{k}, b_{j^{*}}^{*}\left(t_{j^{*}}^{K}\right)\right) \\
& >\left(\frac{f_{\text {low }}}{f_{\text {high }}}\right)^{n-1} \Pi_{j^{*}}\left(t_{j^{*}}^{K}, b_{j^{*}}^{*}\left(t_{j^{*}}^{K}\right)\right) \equiv \triangle>0
\end{aligned}
$$

for all $k>K$, a contradiction of (8).

\section{REFERENCES}

BAJARI, P. (2001): "Comparing Competition and Collusion: A Numerical Approach," Economic Theory, 18, 187-205.

Bikhchandani, S., and J. Riley (1991): "Equilibria in Open Common Value Auctions," Journal of Economic Theory, 53, 101-130.

Hendricks, K., J. Pinkse, and R. Porter (2003): "Empirical Implications of Equilibrium Bidding in First-Price, Symmetric, Common Value Auctions," Review of Economic Studies, 70(1), 115-146.

Kagel, J. (1995): "Auctions: A Survey of Experimental Research," in Handbook of Experimental Economics, ed. by J. Kagel, and A. Roth. Princeton University Press.

Lebrun, B. (1999): "First Price Auctions in the Asymmetric N Bidder Case," International Economic Review, 40(1), 125-142.

Lizzeri, A., And N. Persico (2000): "Uniqueness and Existence of Equilibrium in Auctions with a Reserve Price," Games and Economic Behavior, 30, 83-114. 
Marshall, R., M. Meurer, J.-F. Richard, and W. Stromquist (1994): "Numerical Analysis of Asymmetric First-Price Auctions," Games and Economic Behavior, 7, 193-220.

Maskin, E., And J. Riley (2003): "Uniqueness of Equilibrium in Sealed High-Bid Auctions," Games and Economic Behavior, 45, 395-409.

McAdams, D. (2003): "Characterizing Equilibria in Asymmetric First-Price Auctions," Manuscript, available at http://www.mit.edu/ ${ }^{\sim}$ mcadams.

Milgrom, P. (1981): "Rational Expectations, Information Acquisition, and Competitive Bidding," Econometrica, 49(4), 921-943.

Milgrom, P., And R. Weber (1982): "A Theory of Auctions and Competitive Bidding," Econometrica, 50(5), 1089-1122.

Nalebuff, B., and J. Riley (1985): "Asymmetric Equilibria in the War of Attrition," Journal of Theoretical Biology, 113, 517-527.

Reny, P., And S. Zamir (2004): "On the Existence of Pure Strategy Monotone Equilibria in Asymmetric First-Price Auctions," Econometrica, forthcoming. 